\title{
A note on the Frobenius and the Sylvester numbers
}

\author{
Amitabha Tripathi \\ Department of Mathematics, Indian Institute of Technology \\ Hauz Khas, New Delhi - 110016, India \\ e-mail: atripath@maths.iitd.ac.in
}

Received: 30 March 2017

Accepted: 7 May 2018

\begin{abstract}
Positive integers that cannot be represented by a linear form with relatively prime coefficients and over nonnegative integers are finite in number. We describe a connection between the largest number in this set and the cardinality of this set. We also describe a connection with a subset related to this set.
\end{abstract}

Keywords: Representable, Frobenius number.

2010 Mathematics Subject Classification: 11D07.

The Frobenius Coin Exchange problem revolves around the set $\Gamma^{c}\left(\left\{a_{1}, \ldots, a_{k}\right\}\right)$ of positive integers that are not representable by the linear form $a_{1} x_{1}+\cdots+a_{k} x_{k}$. For brevity, let us call $A=\left\{a_{1}, \ldots, a_{k}\right\}$. For the set $\Gamma^{c}(A)$ to be a finite set it is necessary and sufficient that gcd $A=1$. The Frobenius problem operates under this assumption. Two classical problems involving the set $\Gamma^{c}(A)$ are the determination of the functions $\mathrm{g}(A)$ and $\mathrm{n}(A)$, both due to Sylvester [2], given by

$$
\mathrm{g}(A):=\max \Gamma^{c}(A), \quad \mathrm{n}(A):=\left|\Gamma^{c}(A)\right| \text {. }
$$

The number $\mathrm{g}(A)$ is often called the Frobenius number of $A$ and the number $\mathrm{n}(A)$ sometimes called the Sylvester number of $A$, the former due to the fact that Frobenius popularized the problem posed by Sylvester in his lectures.

The set $\Gamma(A)=\left\{a_{1} x_{1}+\cdots+a_{k} x_{k}: x_{i} \geq 0\right\}$ is closed under addition. So at most one of $n, \mathrm{~g}(A)-n$ can belong to $\Gamma(A)$. By pairing the integers $n$ and $\mathrm{g}(A)-n$ in $\{0, \ldots, \mathrm{g}(A)\}$, we see that at least one integer in each pair belongs to $\Gamma^{c}(A)$. Hence $\mathrm{n}(A) \geq \frac{1}{2}(1+\mathrm{g}(A))$. Equality occurs precisely when exactly one of $n, \mathrm{~g}(A)-n$ belongs to $\Gamma^{c}(A)$, for each $n \in\{0, \ldots, \mathrm{g}(A)\}$. 
This inequality, together with different conditions under which equality may occur, appear in [1]. However, the equivalence we state and prove next is very easy to see and possibly has the status of folklore.

Theorem 1. Let $A$ be a set of positive integers with $\operatorname{gcd} A=1$. The following are equivalent:

(i) $n \in \Gamma^{c}(A)$ implies $\mathrm{g}(A)-n \in \Gamma(A)$ for each $n \in\{0, \ldots, \mathrm{g}(A)\}$.

(ii) $\mathrm{n}(A)=\frac{1}{2}(1+\mathrm{g}(A))$.

Proof. Condition (ii) holds exactly when one of $n, \mathrm{~g}(A)-n$ belongs to $\Gamma(A)$ and the other to $\Gamma^{c}(A)$, for each $n \in\{0, \ldots, \mathrm{g}(A)\}$. Since we already have this situation to hold when $n \in \Gamma(A)$, the remaining condition, given by (i), is equivalent to condition (ii).

The fact that $\Gamma(A)$ is closed under addition implies $n+\Gamma(A) \subseteq \Gamma(A)$ whenever $n \in \Gamma(A)$. What if we asked for the same property to hold for $n \in \Gamma^{c}(A)$ ? We will need to modify the condition a little, since $0 \in \Gamma(A)$ and $n+0 \notin \Gamma(A)$. To exclude this trivial possibility, we define

$$
\mathcal{S}^{\star}(A):=\left\{n \in \Gamma^{c}(A): n+\Gamma^{\star} \subset \Gamma^{\star}\right\}
$$

where $\Gamma^{\star}(A)=\Gamma(A) \backslash\{0\}$.

The set $\mathcal{S}^{\star}(A)$ is never empty, for $\mathrm{g}(A) \in \mathcal{S}^{\star}(A)$. Is it ever possible for $\mathcal{S}^{\star}(A)=\{\mathrm{g}(A)\}$ ? To answer this question, fix $a \in A$, and let $\mathbf{m}_{x}$ denote the smallest integer in $\Gamma(A) \cap(x)$, where $(x)$ denotes the residue class of $x$ modulo $a$. Thus $\Gamma^{c}(A) \cap(x)$ consists of the nonnegative integers of the form $\mathbf{m}_{x}-\lambda a$, with $\lambda \geq 1$. Since $\left(\mathbf{m}_{x}-\lambda a\right)+a \notin \Gamma(A)$ for $\lambda>1$, we have

$$
\mathcal{S}^{\star}(A) \subseteq\left\{\mathbf{m}_{x}-a: 1 \leq x \leq a-1\right\}
$$

In order that $\mathbf{m}_{x}-a \in \mathcal{S}^{\star}(A)$ for some $x \in\{1, \ldots, a-1\}$, it is necessary that $\left(\mathbf{m}_{x}-a\right)+\mathbf{m}_{y} \in$ $\Gamma(A)$ for each $y \in\{1, \ldots, a-1\}$. This condition is also sufficient since any $n \in \Gamma(A)$ is of the form $\mathbf{m}_{y}+\lambda a$ with $y \in\{0, \ldots, a-1\}$ and $\lambda \geq 1$. Since $\left(\mathbf{m}_{x}-a\right)+\mathbf{m}_{y} \equiv x+y \bmod a$, we must have $\left(\mathbf{m}_{x}-a\right)+\mathbf{m}_{y} \geq \mathbf{m}_{x+y}$, for each $y \in\{1, \ldots, a-1\}$. Hence we have shown that

$$
\mathbf{m}_{x}-a \in \mathcal{S}^{\star}(A) \Longleftrightarrow \mathbf{m}_{x}+\mathbf{m}_{y} \geq \mathbf{m}_{x+y}+a \text { for } 1 \leq y \leq a-1 .
$$

The definition in Eqn. (2) and results in Eqn. (3) and Eqn. (4) are from [3].

We are now in position to partially answer the question about when $\mathcal{S}^{\star}(A)=\{\mathrm{g}(a, b)\}$. The connection is due to the fact that the largest integer in $\Gamma^{c}(A)$ is the largest among $\mathbf{m}_{x}-a$, with $x \in\{1, \ldots, a-1\}$.

Theorem 2. Let $A$ be a set of positive integers with $\operatorname{gcd} A=1$. If $n \in \Gamma^{c}(A)$ implies $\mathrm{g}(A)-n \in$ $\Gamma(A)$ for each $n \in\{0, \ldots, \mathrm{g}(A)\}$, then

$$
\mathcal{S}^{\star}(A)=\{\mathrm{g}(A)\}
$$


Proof. Note that $\mathrm{g}(A) \in \mathcal{S}^{\star}(A)$ because any integer greater than $\mathrm{g}(A)$ belongs to $\Gamma(A)$. Fix $a \in A$, and let $\mathrm{g}(A)=\mathbf{m}_{r}-a$ with $r \in\{1, \ldots, a-1\}$.

Suppose condition (i) of Theorem 1 holds. Then exactly one of $n, \mathrm{~g}(A)-n$ belongs to $\Gamma^{c}(A)$, for each $n \in\{0, \ldots, \mathrm{g}(A)\}$. Suppose $n \in \mathcal{S}^{\star}(A), n \neq \mathrm{g}(A)$. Then $n=\mathbf{m}_{x}-a$ for some $x \in\{1, \ldots, a-1\} \backslash\{r\}$. Since $n \in \Gamma^{c}(A), \mathrm{g}(A)-n=\mathbf{m}_{r}-\mathbf{m}_{x} \in \Gamma(A)$, and must therefore be at least as much as the least integer in $\Gamma(A)$ in its congruence class. Therefore $\mathbf{m}_{r}-\mathbf{m}_{x} \geq \mathbf{m}_{r-x}$, so that $\mathbf{m}_{x}+\mathbf{m}_{r-x} \leq \mathbf{m}_{r}$. It follows from (4) that $n=\mathbf{m}_{x}-a \notin \mathcal{S}^{\star}(A)$ for $x \neq r$.

Corollary 1. Let $A$ be a set of positive integers with $\operatorname{gcd} A=1$. Then

$$
\mathrm{n}(A)=\frac{1}{2}(1+\mathrm{g}(A)) \text { implies } \mathcal{S}^{\star}(A)=\{\mathrm{g}(A)\} .
$$

There are many instances where the converse of Theorem 2 (or to Corollary 1 ) holds. One such instance is the case of the geometric sequence $A=\left\{a^{k}, a^{k-1} b, \ldots, b^{k}\right\}$, where $\operatorname{gcd}(a, b)=1$. However, for the arithmetic sequence $A=\{a, a+d, \ldots, a+k d\}$, where $\operatorname{gcd}(a, d)=1$, it turns out that whereas $\mathrm{n}(A)>\frac{1}{2}(1+\mathrm{g}(A))$, we have $\mathcal{S}^{\star}(A)=\{\mathrm{g}(A)\}$ when $k \mid(a-2)$; refer [3].

\section{References}

[1] Nijenhuis, M. \& Wilf, H. S. (1972) Representation of integers by linear forms in nonnegative integers, J. Number Theory, 4, 98-106.

[2] Sylvester, J. J. (1884) Problem 7382, in W. J. C. Miller, ed., Mathematical Questions, with their Solutions, from the "Educational Times", 41, 1884, p. 21. Solution by W. J. Curran Sharp.

[3] Tripathi, A. (2003) On a variation of the Coin Exchange Problem for Arithmetic Progressions, Integers, 3, Article A01, 5 pages. 